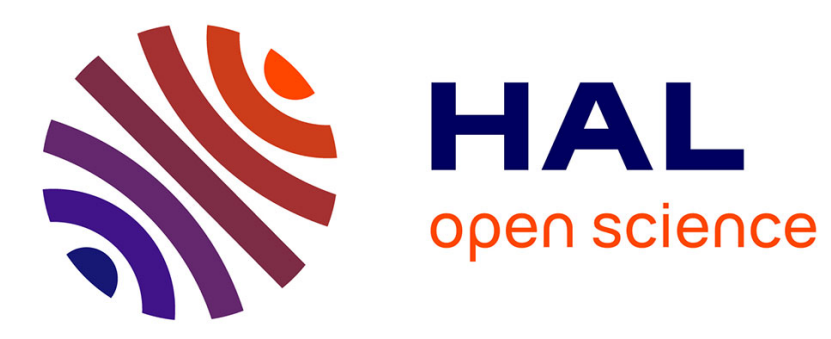

\title{
Influence of thin inversion layers on Schottky diodes K.K. Sharma
}

\section{To cite this version:}

K.K. Sharma. Influence of thin inversion layers on Schottky diodes. Revue de Physique Appliquée, 1986, 21 (1), pp.25-33. 10.1051/rphysap:0198600210102500 . jpa-00245407

\section{HAL Id: jpa-00245407 https://hal.science/jpa-00245407}

Submitted on 1 Jan 1986

HAL is a multi-disciplinary open access archive for the deposit and dissemination of scientific research documents, whether they are published or not. The documents may come from teaching and research institutions in France or abroad, or from public or private research centers.
L'archive ouverte pluridisciplinaire HAL, est destinée au dépôt et à la diffusion de documents scientifiques de niveau recherche, publiés ou non, émanant des établissements d'enseignement et de recherche français ou étrangers, des laboratoires publics ou privés. 
Classification

Physics Abstracts

$73.40 \mathrm{~N}$

\title{
Influence of thin inversion layers on Schottky diodes
}

\author{
K. K. Sharma $(*)$ \\ Laboratoire de Physique des Solides, CNRS, 1 pl. A. Briand, F-92190 Meudon, France
}

(Reçu le 28 mai 1985, accepté le 16 septembre 1985)

\begin{abstract}
Résumé. - Une diode Schottky voit sa hauteur de barrière augmenter lorsque le dopage du semiconducteur est inversé en surface. Cet effet est calculé, de façon à mettre en évidence l'influence des principaux paramètres : dopage et épaisseur de la couche d'inversion, configuration des états d'interface, fonction de travail du métal. L'interprétation des rares résultats expérimentaux publiés permet de conclure que les états d'interface ne sont pas modifiés par la présence de la couche d'inversion.
\end{abstract}

Abstract. - A Schottky diode exhibits a barrier height which increases when the semiconductor doping is superficially inversed. This effect is modellized, so as to show the influence of the main parameters : doping and thickness of the inversion layer, interface state configuration, metal work function. The analysis of a few available experimental results is consistent with the assumption that the interface states are not modified by the inversion layer.

\section{Introduction.}

The properties of metal-semiconductor (MS) junctions depend on the detailed electrical and chemical nature of the interface between both materials. While surface science techniques give best insight into this interface, as reviewed recently by Brillson [1], it is also helpful to use a more macroscopic approach and relate the junction properties to the proper physical parameters. Among the latter, most important are the interfacial electronic states and the values of work functions of the metal $\phi_{\mathrm{m}}$ and the bulk semiconductor $\phi_{\mathrm{s}}$. The difference of $\phi_{\mathrm{m}}$ and $\phi_{\mathrm{s}}$ may cause a band bending to occur at the semiconductor surface, due to a charge transfer which is necessary to equalize the Fermi level in both sides when they are put in contact, upon equilibrium conditions; however this effect was shown 20 years ago to be unable to explain the experimentally observed barrier heights, which turn out to be little dependent on metal work function. In order to explain this behaviour, Cowley and Sze [2], using a former idea of Bardeen [3], described the interface as a dipole layer and, introducing the interface states as essentially responsible for the band bending (which remains parabolic) could account for the barrier heights quantitatively. The complete distribution of interface states throughout the energy gap of the semiconductor

(*) Detached from R.K. College Shamli, Univ. of Meerut, India. is not needed for this purpose; the model can be built on the knowledge of $E_{\mathrm{N}}$, the level up to which the surface states have to be filled to ensure charge neutrality of the semiconductor surface, and of $D_{\mathrm{s}}$, the density of surface states near this level, expressed in $\mathrm{cm}^{-2} \mathrm{eV}^{-1}$. More recent knowledge of the interface state distribution may somewhat modify the model, as suggested by Barret and Vapaille [4].

If the semiconductor is not uniformly doped, its doping profile below the MS interface introduces a non-parabolicity of the band bending and may change largely the barrier height $\phi_{\mathrm{b}}$ and other properties of the diode. This may happen naturally due to some metal-semiconductor reaction [1] or be deliberately produced. For instance Card [5, 6], using an alloying process between $\mathrm{Al}$ and $\mathrm{n}-\mathrm{Si}$, could raise $\phi_{\mathrm{b}}$ from $0.66 \mathrm{eV}$ to $0.74 \mathrm{eV}$. Also Li et al. [7] implanted phosphorous on $\mathrm{p}$-Si before building a Ti-Si Schottky diode, thus increasing $\phi_{\mathrm{b}}$ from $0.6 \mathrm{eV}$ to $0.96 \mathrm{eV}$. Qualitative explanations of these results were given, using the likely trends of the band structure due to the inversion layer. Similar results were found for AsGa-based Schottky diodes [8].

We present here a model which aims at : i) describing simple Schottky diodes - and in this case, integrating to Cowley and Sze's theory recent knowledge on interface states, and also illustrating the role of the most important physical parameters - and ii) covering the case when a compensating or even an inverted layer has been introduced by proper 
doping of the semiconductor surface before depositing the metal. In this first step, we are interested only in barrier heights, though electrical and photoelectric properties might be studied later on, and we choose silicon as the test material to illustrate the model.

\section{Summary of the model for simple Schottky diodes.}

Following Cowley and Sze [2] we shall use the following assumptions :

(i) There exists an intimate contact between metal and semiconductor with an interfacial layer of atomic dimensions (say $5 \AA$ ), which is transparent to electrons and holes. The interfacial layer can withstand the potential across it and its permittivity $\varepsilon_{\mathrm{i}}$ is that of free space $\varepsilon_{0}$.

(ii) Interfacial states exist between the semiconductor and the interfacial layer; their density vs. energy is essentially characteristic of the semiconductor; their occupation probability depends on the metal and semiconductor work functions.

(iii) There is negligible barrier lowering by Schottky image effect.

Let the energy band configuration of a metal-n type semiconductor system be as shown by figure $1, \phi_{m}$ is the metal work function and $\phi_{\mathrm{s}}$ that of the semiconductor. The electron affinity of the latter is $\chi=\phi_{\mathrm{s}}-V_{\mathrm{n}}$, where $V_{\mathrm{n}}$ is a well-known function of the doping and of the conduction band structure [9]. $\phi_{\text {bn }}$ is the barrier height which is surmounted by the electrons flowing from the metal to semiconductor side. The neutral level, up to which the interface states are filled before the formation of the diode, has an energy difference $\phi_{0}$ with the valence band edge and $\phi_{\mathrm{g}}$ with the Fermi level $E_{\mathrm{F}} . W_{\mathrm{n}}$ is the thickness of the depleted n-region and $\delta$, the thickness of the interfacial

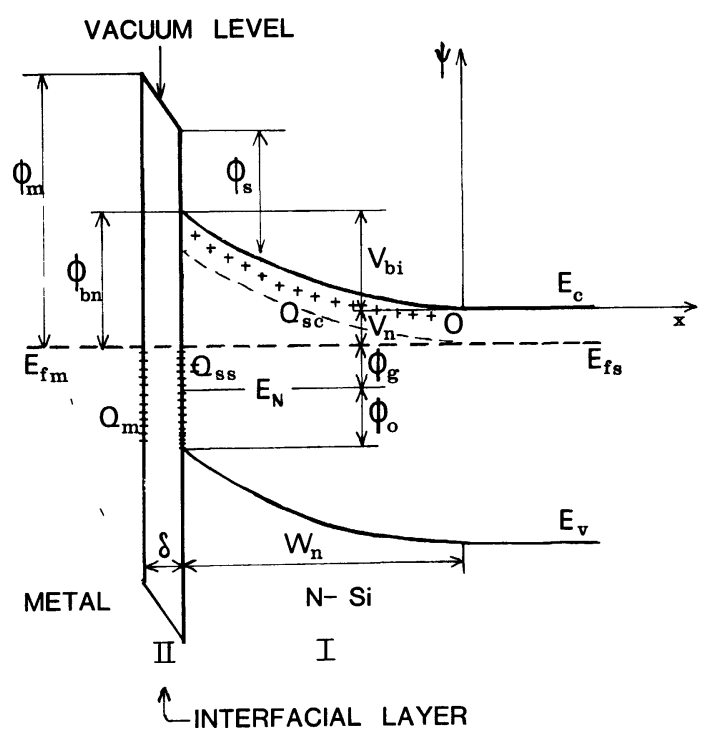

Fig. 1. - Schematic representation of a simple Schottky diode $\mathrm{metal} / \mathrm{n}-\mathrm{Si}$. layer. The abscissa $x$ will be counted from the onset of the band bending, inside the semiconductor. The zero potential will be chosen to be that of electrons at level $E_{\mathrm{c}}$.

We can know the exact energy band diagram by solving the Poisson's equation in different regions and then applying the condition of electronic equilibrium. In region I, the band edges exhibit the classical parabolic behaviour of a depleted region. In region II, the potential fall $\Delta$ is related by Gauss's law to the charge of the dipole layer. Two types of charges are present on the semiconductor side of this layer : those transferred from the ions of the depleted region, the density of which is :

$$
Q_{\mathrm{sc}}=q N_{\mathrm{D}} W_{\mathrm{n}} \text { Coulomb } / \mathrm{cm}^{2}
$$

( $q$ = electron charge, $N_{\mathrm{d}}=$ density of ionized donors in the bulk semiconductor) and those due to interface states. The density of the latter, $Q_{\text {ss }}$, will be expressed as :

$$
Q_{\mathrm{ss}}=-q D_{\mathrm{s}} \phi_{\mathrm{g}} \text { Coulomb } / \mathrm{cm}^{2}
$$

where $D_{\mathrm{s}}$ is the density of the interface states per unit area per unit electronvolt, assumed to be constant in the energy interval between $E_{\mathrm{N}}$ and $E_{\mathrm{F}}$. One may also write :

$$
Q_{\mathrm{ss}}=-q D_{\mathrm{s}}\left(E_{\mathrm{g}}-\phi_{0}-\phi_{\mathrm{bn}}\right) .
$$

As a counterpart to $Q_{\text {ss }}+Q_{\text {sc }}$, an equal and opposite charge $Q_{\mathrm{M}}$ is developing on the metal side of the interfacial layer :

$$
Q_{\mathrm{M}}=-\left(Q_{\mathrm{ss}}+Q_{\mathrm{sc}}\right) .
$$

$Q_{\text {sc }}$ is at least one order of magnitude smaller than $Q_{\mathrm{ss}}$, in most cases : for $\phi_{\mathrm{bn}}=0.6-0.8 \mathrm{eV}$, equation (1) gives $W_{\mathrm{n}}$ near $1 \mu \mathrm{m}$, so that $Q_{\mathrm{sc}} / q=10^{11} \mathrm{~cm}^{-2}$ for a doping $N_{\mathrm{D}}=10^{15} \mathrm{~cm}^{-3}$; on the other hand we shall see that $D_{\mathrm{s}}$ is of order $10^{13} \mathrm{~cm}^{2} . \mathrm{eV}^{-1}$ and $\phi_{\mathrm{g}}$ is 0.1 to $0.4 \mathrm{eV}$, so that $Q_{\mathrm{ss}} / q$ is higher than $10^{12} \mathrm{~cm}^{-2}$. We may therefore neglect $Q_{\mathrm{sc}}$ before $Q_{\mathrm{ss}}$.

Following Cowley and Sze [2], the equation expressing the electronic equilibrium throughout the structure can be written :

$$
\phi_{\mathrm{bn}}=K\left(\phi_{\mathrm{m}}-\chi\right)+(1-K)\left(E_{\mathrm{g}}-\phi_{0}\right)
$$

where :

$$
K=\frac{\varepsilon_{\mathrm{i}}}{\varepsilon_{\mathrm{i}}+q \delta D_{\mathrm{s}}} .
$$

The barrier height $\phi_{\mathrm{bn}}$ behaves differently according to the value of $D_{\mathrm{s}}$ :

- if $D_{\mathrm{s}}=0, \phi_{\mathrm{bn}}=\phi_{\mathrm{m}}-\chi$, which is the barrier height in the frame of the simple Schottky theory;

- if $D_{\mathrm{s}} \rightarrow \infty, \phi_{\mathrm{bn}}=E_{\mathrm{g}}-\phi_{0}$, which means that the Fermi level is pinned with the neutral level; in this case the barrier height is independent of $\phi_{\mathrm{m}}$; 
- in reality, $D_{\mathbf{s}}$ lies somewhere between these two extremes; if it is furthermore assumed to be a constant for a given semiconductor and all metals, $\phi_{b n}$ is found to be a linear function of the metal work function $\phi_{\mathrm{m}}$.

\section{Results and discussion for simple Schottky diodes.}

We shall illustrate the variations of barrier height with the physical parameters involved, with the help of figures 2 to 5 .

Figure 2 shows, for Au-nSi system, that $\phi_{\mathrm{b}}$ is quite independent of the semiconductor doping. This is because the barrier height is the sum of two terms : the built-in potential $V_{b i}$, which increases with the doping density $N_{\mathrm{d}}$, and the term $V_{\mathrm{n}}$, which decreases by the same amount. Experiments have confirmed this finding, which is coherent with the fact that $Q_{\mathrm{sc}} \ll Q_{\mathrm{ss}}$.

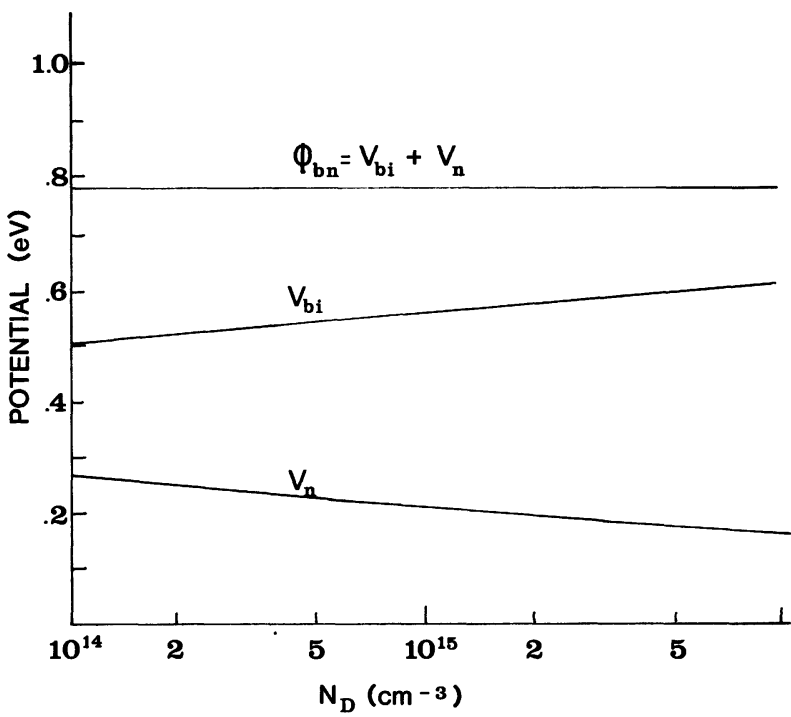

Fig. 2. - Barrier height as a function of doping for Au-nSi system with $D_{\mathrm{S}}=4 \times 10^{13}$ states $/ \mathrm{cm}^{2}$.eV.

Figure 3 shows the influence of surface state density on barrier height, for various diodes based on $\mathrm{n}-\mathrm{Si}$. $\phi_{\mathrm{b}}$ increases with $D_{\mathrm{s}}$ for low values of $D_{\mathrm{s}}$, and this increment is more prominent in the case of low work function metals. However, towards large densities of surface states $\left(\simeq 10^{15} \mathrm{~cm}^{-2} . \mathrm{eV}^{-1}\right)$ the barrier height becomes fixed and independent both on $D_{\mathbf{s}}$ and on metal work function. This is the case where the Fermi level has been pinned at the neutral level, so that the barrier height is almost equal to $E_{\mathrm{C}}-E_{\mathrm{N}}$ : $\phi_{\mathrm{b}}=E_{\mathrm{g}}-\phi_{0}$.

Figure 4 shows clearly that the distance between Fermi level and neutral level decreases when the density of surface states increases. For the highest densities of surface states, the Fermi level locks with the neutral level.

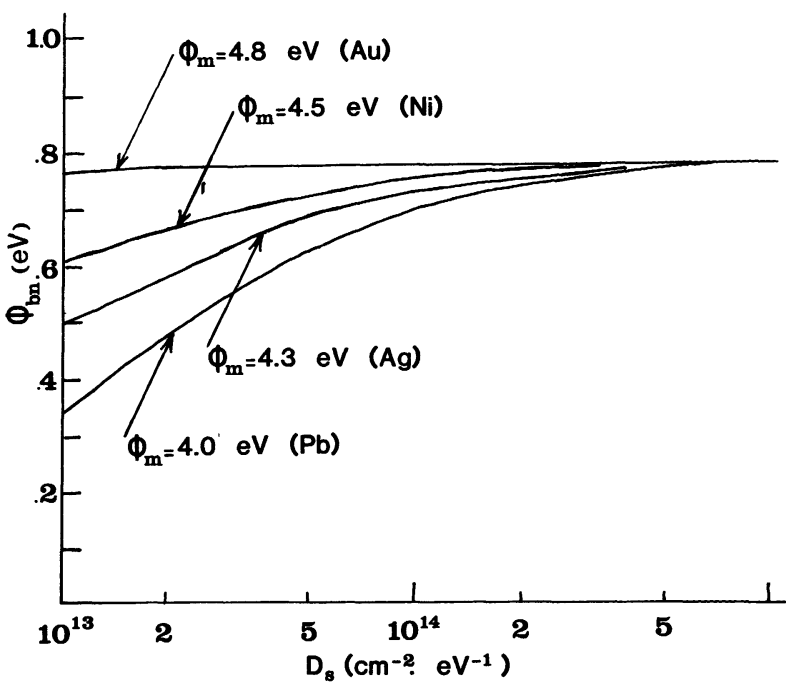

Fig. 3. - Barrier height as a function of interface state density and metal work function $\left(\phi_{0}=0.33 \mathrm{eV}, N_{\mathrm{D}}=\right.$ $10^{15} \mathrm{~cm}^{-3}$ ).

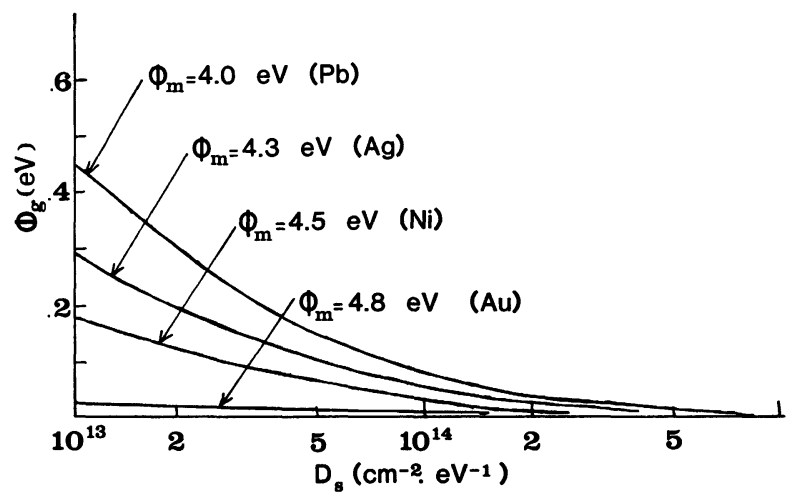

Fig. 4. - Energy difference between Fermi level and neutral level as a function of interface state density and metal work function.

Equation (5) showed that the barrier height depends not only on the density of surface states $D_{\mathbf{s}}$ (through $K$ ), but also on $\phi_{0}$ which appears explicitly. How both $D_{\mathrm{s}}$ and $\phi_{0}$ influence $\phi_{\mathrm{bn}}$ is illustrated by figure 5 . However $D_{\mathrm{s}}$ and $\phi_{0}$ are dependent on the properties of the interface and our discussion will bear first on their values.

In its first version [2], this model was compared to experiments, and found to explain approximately the values of $\phi_{\mathrm{bn}}$ for many metals on $\mathrm{Si}$, provided that the following values of the parameters were chosen (Fig. 6) :

$$
\phi_{0}=0.33 \mathrm{eV} \quad D_{\mathrm{s}}=4 \times 10^{13} \mathrm{~cm}^{-2} \cdot \mathrm{eV}^{-1} .
$$

Figure 6 shows that these values fit well the cases of a series of metals $(\mathrm{Pb}, \mathrm{Al}, \mathrm{Ag}, \mathrm{Cu}, \mathrm{Au})$ having barrier heights, on $\mathrm{n}-\mathrm{Si}$, ranging from $0.6 \mathrm{eV}$ to $0.8 \mathrm{eV}$ (experimental points are from Milnes [10]). However other 


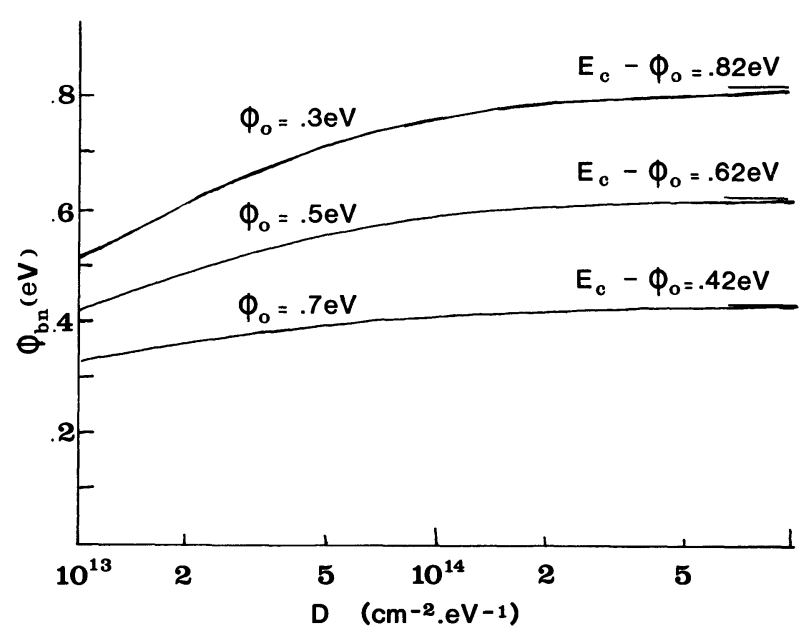

Fig. 5. - Barrier height as a function of $D_{\mathrm{S}}$ and $\phi_{0}$ for $\mathrm{Ag} / \mathrm{n}-\mathrm{Si}$ Schottky diodes.

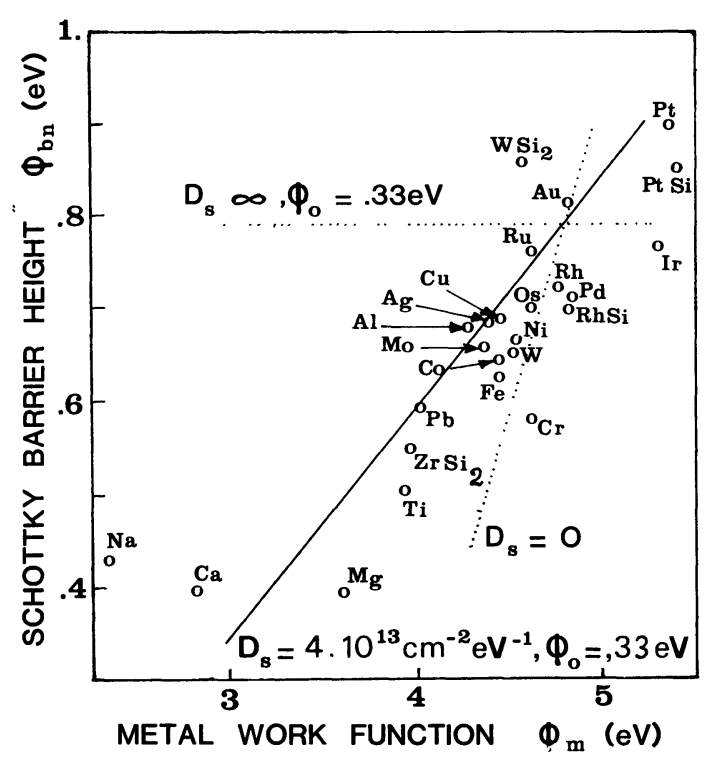

Fig. 6. - Experimental determinations of barrier heights vs. metal work functions (Ref. [10]). The straight lines represent theoretical variations for different values of $D_{s}, \phi_{0}$.

metals do not fit (12). Our model is able to explain, at least semiquantitatively, the different cases seen on figure 6 , as follows.

$\phi_{0}$ is a property of the semiconductor which is decided by the neutrality of its surface before evaporating the metal over it : it should be essentially constant for a given semiconductor in contact with any metal, though variations according to crystal orientation or surface bond reconstruction may happen to take place. The best approximation, as mentioned above, is to choose this constant equal to $0.33 \mathrm{eV}$. On contrary, the number of surface states per unit area, $D_{\mathbf{s}}$, may change drastically from metal to metal. $D_{s}$ is the mean density of states in the interval, of width $\phi_{\mathrm{g}}$, between neutrality level and equilibrium Fermi level. How the density of interface states varies inside the energy gap results from several recent works $[4,11,12]:$ it is admitted that $D_{\mathrm{s}}(E)$ is maximal near the valence band and conduction band, and minimal in the middle of the gap. Now let us consider the cases of $\mathrm{Al}, \mathrm{Cr}$ and $\mathrm{Na}$. Using the values of $\phi_{\mathrm{m}}$ and $\phi_{\mathrm{bn}}$ illustrated by figure 6 and those of the constants : $\chi=4.05 \mathrm{eV}, \phi_{0}=0.33 \mathrm{eV}, K$ can be found from formula (5), and then $D_{s}$ from formula (6). On the other hand $\phi_{\mathrm{g}}$ is equal to $E_{\mathrm{g}}-\phi_{0}-\phi_{\mathrm{bn}}$. The results are :

$$
\begin{array}{ll}
\mathrm{Al}: \phi_{\mathrm{g}}=0.11 \mathrm{eV} ; & D_{\mathrm{s}}=5 \times 10^{13} \mathrm{~cm}^{-2} \mathrm{eV}^{-1} \\
\mathrm{Cr}: \phi_{\mathrm{g}}=0.21 \mathrm{eV} ; & D_{\mathrm{s}}=6 \times 10^{11} \mathrm{~cm}^{-2} \mathrm{eV}^{-1} \\
\mathrm{Na}: \phi_{\mathrm{g}}=0.56 \mathrm{eV} ; & D_{\mathrm{s}}=7.5 \times 10^{13} \mathrm{~cm}^{-2} \mathrm{eV}^{-1}
\end{array}
$$

These results can be compared to the known energy variation of the interface state density, as given for instance by figure 2 of Barret-Vapaille's paper [4]. This work applies to $\mathrm{Cr}$-Si Schottky diodes prepared by ultra-vacuum evaporation of $\mathrm{Cr}$ on cleaved $\mathrm{Si}$ surfaces, i.e. to very clean $\mathrm{Cr} / \mathrm{Si}$ interfaces : in the region from 0.33 to 0.54 above valence band, $D_{\mathrm{s}}$ is minimal, of order $5 \times 10^{11} \mathrm{~cm}^{-2} \mathrm{eV}^{-1}:$ this is exactly the value that was derived above from our model. No such quantitative agreement can be obtained for $\mathrm{Al}$ or $\mathrm{Na}$, because in these cases no direct results about $D_{\mathrm{s}}$ have been obtained from cleaved surfaces; however it is clear from the general shape of the $D_{\mathrm{s}}(E)$ curve that, when $\phi_{\mathrm{g}}$ decreases from that of $\mathrm{Cr}$ to that of $\mathrm{Al}, D_{\mathrm{s}}$ should increase since the Fermi level gets nearer the "A-band " of reference [4] (near the valence band), whereas, when $\phi_{\mathrm{g}}$ increases from that of $\mathrm{Cr}$ to that of $\mathrm{Na}, D_{\mathrm{s}}$ should also increase since the Fermi level gets nearer the "C-band" of reference [4] (near the conduction band) : it is exactly the trend found above from our model. This nice semiquantitative fit justifies assumption (ii) of $\S 2$.

One could argue that our value $\varepsilon_{\mathrm{i}}=\varepsilon_{0}$ (assumption (i)) is arbitrary. But if we try $\varepsilon_{i}=3.9 \varepsilon_{0}$ instead, a value typical of silicon oxide, we will find $D_{\mathrm{s}} \delta$ to be 3.9 times larger. Since the density of $\mathrm{Si}_{-} \mathrm{SiO}_{2}$ interface states has been consistently found to be smaller than $10^{13} \mathrm{~cm}^{-2} \mathrm{eV}^{-1}[11,12], \delta$ should be of order of several tens of $\AA$. This is not the case in the structures which we are studying, in which no oxide layer has been deliberately grown between the semiconductor and the metal.

As for our assumption (iii), the neglect of the barrier lowering due to the image force effect is justified, since this effect does not contribute more than $0.02 \mathrm{eV}$ to the energy band configuration.

We can conclude that numerical values like (7) are approximately valid for $\mathrm{Al}$ and similar metals, and that divergencies from these values.for some other metals are qualitatively accounted for. This discussion refers to " extrinsic " surface states (after the metal has been deposited). The model thus built for simple diodes has now to be extended to $n / p$ metal (or $p / n$ metal) structures. 


\section{Model for diodes with inversion layers.}

Our approach will allow to go further than the preceding tentative of Van der Ziel [13], into the case of Schottky diodes having an inversion layer between the bulk semiconductor and the metal. Figure 7 illustrates the case of a highly doped inversion layer, of thickness $W_{\mathbf{p}}$.

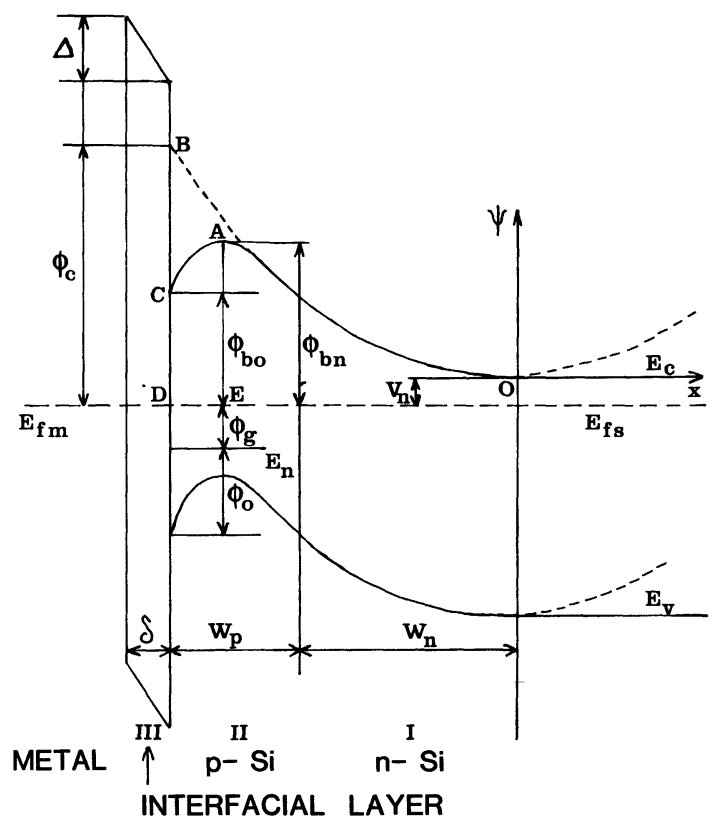

Fig. 7. - Schematic representation of a metal $/ \mathrm{p} / \mathrm{Si} / \mathrm{n} / \mathrm{Si}$ Schottky diode.

The three initial assumptions of $\S 2$ will be made again, together with two other assumptions :

(iv) The surface states at the metal/p-Si interface are the same as they were, in $\S 2$, for metal/n-Si interfaces.

(v) The inversion layer is abrupt, thin and fully ionized.

In region I, we still have parabolic bands. In region II, the solution of Poisson's equation, using proper boundary conditions, is found to be :

$$
\Psi=\frac{q N_{\mathrm{a}}}{\varepsilon_{\mathrm{s}} \varepsilon_{0}} \frac{x^{2}}{2}+C x+D
$$

with :

$$
C=-\frac{q\left(N_{\mathrm{d}}+N_{\mathrm{a}}\right)}{\varepsilon_{\mathrm{s}} \varepsilon_{0}} W_{\mathrm{n}}
$$

and :

$$
D=-\frac{q N_{\mathrm{d}}}{\varepsilon_{0} \varepsilon_{\mathrm{s}}} \frac{W_{\mathrm{n}}^{2}}{2}-\frac{q N_{\mathrm{a}}}{\varepsilon_{0} \varepsilon_{\mathrm{s}}} \frac{W_{\mathrm{n}}^{2}}{2}-C W_{\mathrm{n}} .
$$

We thus find the potential at the end of region II, $(\Psi)_{x=W_{n}}+W_{p}$.
To know the potential drop $\Delta$ at the interfacial layer, one needs to know the total surface charge at the semiconductor surface. There are three contributions to this charge :

(i) From the ionized p-region

(ii) From the ionized $n$-region

(iii) From the surface states.

Let the contribution by $\mathrm{n}$ and $\mathrm{p}$ regions be denoted by $Q_{\text {sc }}$ and that contributed by surface states by $Q_{\text {ss }}$. Then,

$$
Q_{\mathrm{sc}}=-q N_{\mathrm{a}} W_{\mathrm{p}}+q N_{\mathrm{d}} W_{\mathrm{n}} \text { Coulomb } / \mathrm{cm}^{2}
$$

and

$$
Q_{\mathrm{ss}}=-q D_{\mathrm{s}} \phi_{\mathrm{g}} \text { Coulomb } / \mathrm{cm}^{2}
$$

by the definition of the neutral level.

Applying Gauss's law at the interface gives $\Delta$, so that we now know :

$$
(\Psi)_{x=W_{n}+W_{p}+\delta}=(\Psi)_{x=W_{n}+W_{p}}+\Delta .
$$

Finally the electronic equilibrium requires that the latter potential be equal to $\phi_{\mathrm{m}}-\phi_{\mathrm{s}}$. This helps to find out the potential distribution at the various points of the device in thermal equilibrium, and in particular the effective barrier height.

If the potential (8) in region II is such that, $N_{\mathrm{a}} W_{\mathrm{p}}>N_{\mathrm{d}} W_{\mathrm{n}}$, there exists a maximum potential point in the p-region. Obviously, the effective barrier height of the device will be equal to the height of this point above the Fermi level. The position $x_{\mathrm{m}}$ and height $\phi_{\mathrm{bn}}$ of this maximum potential point are found to be :

$$
\begin{gathered}
x_{\mathrm{m}}=-\frac{C \varepsilon_{\mathrm{s}} \varepsilon_{0}}{q N_{\mathrm{a}}}=\frac{\left(N_{\mathrm{d}}+N_{\mathrm{a}}\right) W_{\mathrm{n}}}{N_{\mathrm{a}}} \\
\phi_{\mathrm{bn}}=\left(D-\frac{C^{2} \varepsilon_{\mathrm{s}} \varepsilon_{0}}{2 q N_{\mathrm{a}}}\right)+V_{\mathrm{n}} .
\end{gathered}
$$

\section{Results and discussion for $n / p$ metal diodes.}

Following the above analysis, computerized calculation has been made to understand the various properties of metal-p-nSi devices. However, the model is general and can be applied to any such semiconductor device. The results are shown from figure 8 to 14 .

Figure 8 shows the effect of introducting a thin surface layer on the Schottky diodes of different metals. It has been found that the barrier height versus metal workfunction curves, for fixed widths and doping densities of the surface layer, are straight lines. The increment in barrier height due to a particular surface layer is independent of the metal workfunction.

Figure 9 shows the effect of substrate doping on the barrier height of a silver-p-n silicon system. Unlike Schottky barriers (Fig. 2), the barrier height in such devices is very sensitive to the doping density of the bulk semiconductor. It decreases when $N_{\mathrm{d}}$ increases. 


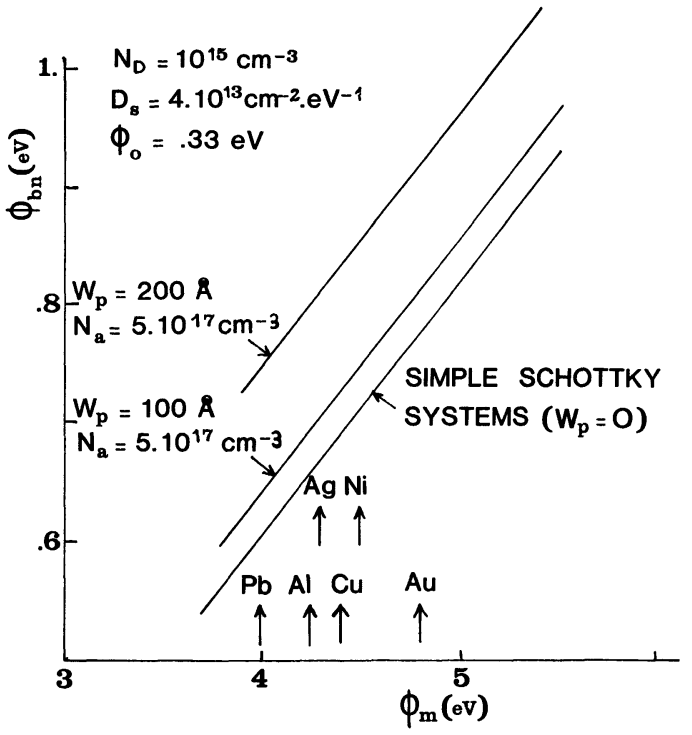

Fig. 8. - Effect of introduction of thin surface layers (of thickness $W_{\mathrm{p}}$, doping $N_{\mathrm{a}}$ ) on various Schottky diodes.

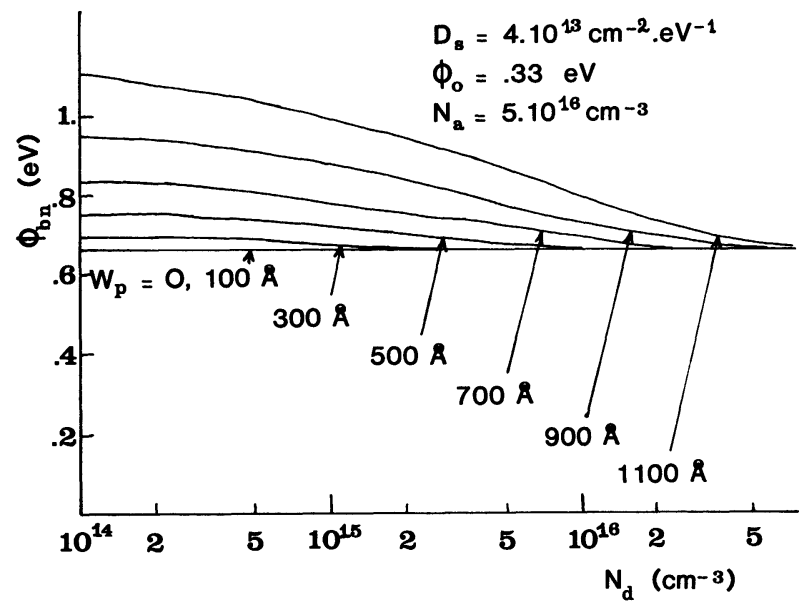

Fig. 9. - Variation of effective barrier height of Ag-p-nSi systems with the doping density of the substrate.

Therefore the less impure semiconductors are the better choice to fabricate such devices.

Figure 10 shows the variation of the effective barrier height with the ionized acceptor density in p-region. Similarly, the variation of barrier height with the thickness of the p-region for its various doping concentrations has been shown in figures 11 and 12 for Ag-p$\mathrm{nSi}$ and $\mathrm{Al}-\mathrm{p}-\mathrm{nSi}$ devices. It has been found that the width and the doping density are the main parameters which determine the barrier height in such devices. However, the surface neutrality conditions can also affect the barrier height formation as shown in figure $13:$ the increase in surface state density at the interface increases the barrier height in such devices. Figure 14 shows the energy band diagram of a peculiar Ag-p-nSi device of barrier height $\sim 0.90 \mathrm{eV}$ based upon the

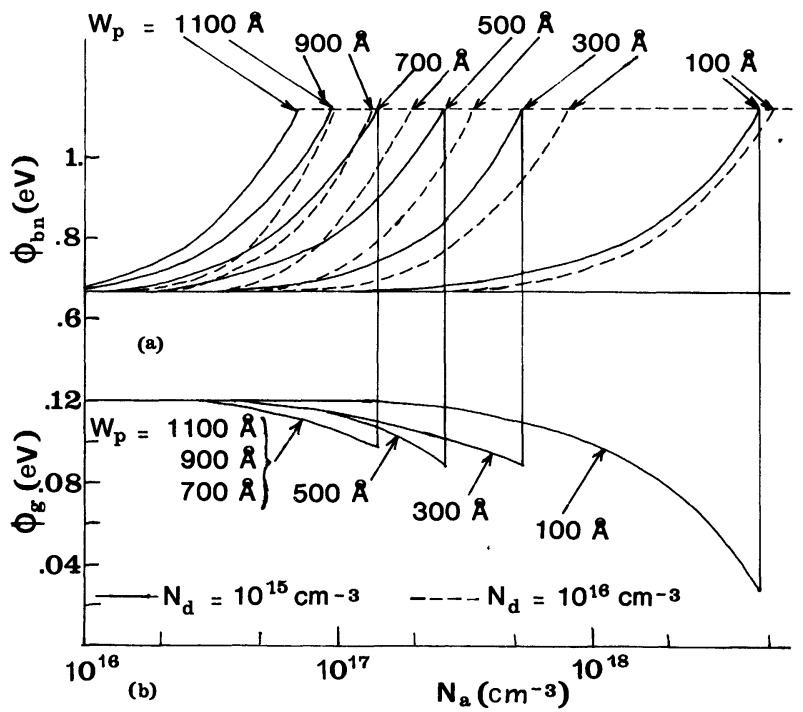

Fig. 10. - (a) Variation of effective barrier height of Ag-p$\mathrm{nSi}$ systems with the density of the ionized acceptors in the p-region for its various widths. (b) Variation of the position of Fermi level relative to neutral level in Ag-p-nSi devices.

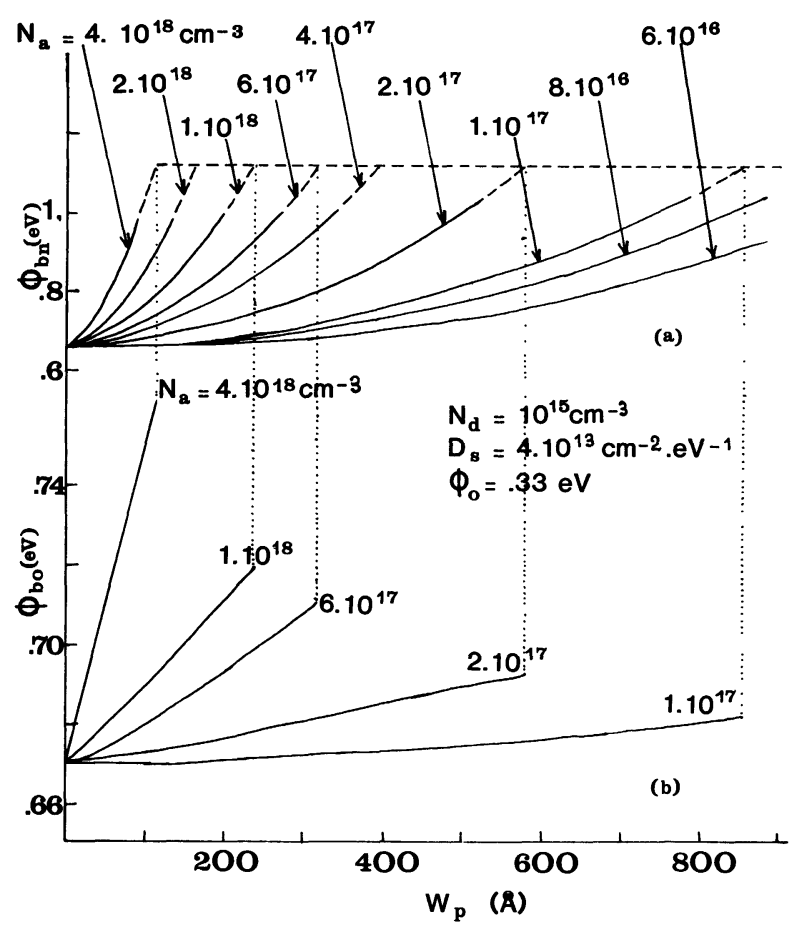

Fig. 11. - (a) Variation of effective barrier height of Ag-p$\mathrm{nSi}$ systems with the thickness of inversion region for its different doping densities. (b) Variation of $\phi_{\mathrm{bo}}$ (see Fig. 7) with the width and doping of the inversion region.

present model, together with that of the corresponding simple diode, without the p-region.

In figure 10, the energy difference between the neutral level and the Fermi level has also been plotted against the doping density of the inversion layer till the barrier height becomes equal to the energy gap. 


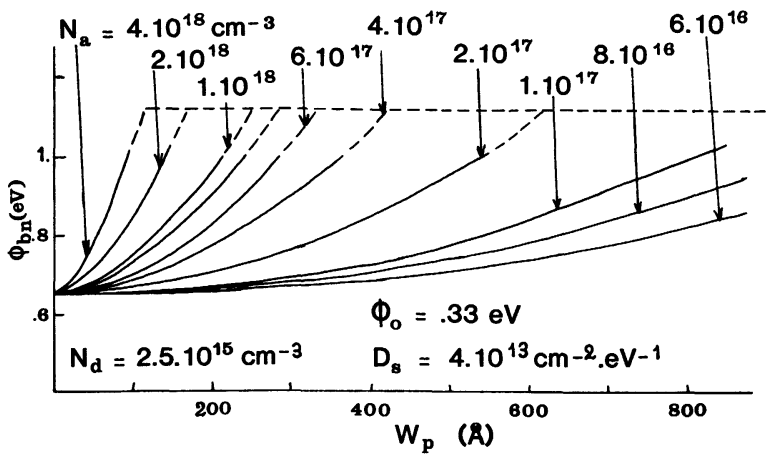

Fig. 12. - Variation of effective barrier height of Al-p-nSi systems with the thickness of p-region for its different doping densities (theory applicable to Card's experiments [5])

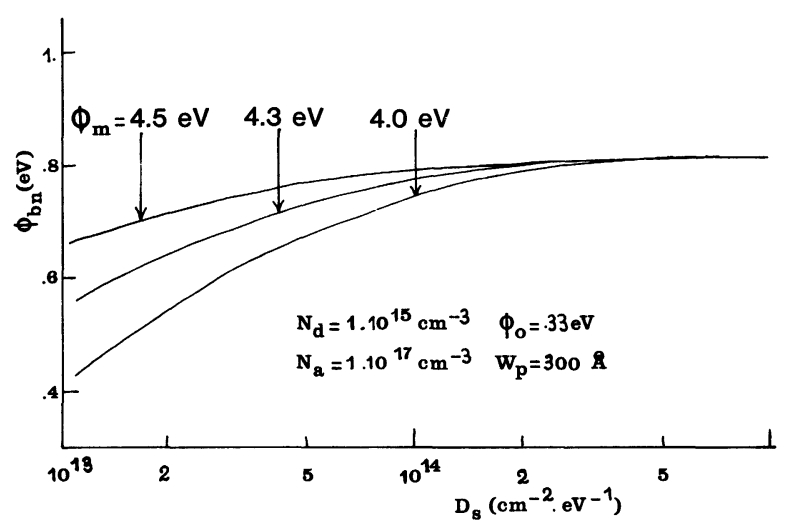

Fig. 13. - The effect of surface state density on the barrier height of different Schottky diodes with inversion layers.

It is seen that towards the larger widths of the surface layer, the difference $\phi_{\mathrm{g}}$ decreases. This parameter gives an estimation of the stability of these devices with respect to time or aging. The aging is the main drawback of Schottky barriers, which is due to the change in surface states at the interface, causing a change in $Q_{\mathrm{ss}}$, i.e. a change of $\phi_{\mathrm{g}} . D_{\mathrm{s}}$ (see Eq. (12)) and a change in barrier height. With metal-p-nSi devices, $\phi_{\mathrm{g}}$ is smaller and changes less with exposition to atmosphere, so that these devices should be more stable.

The potential at the end of p-region at the surface has been depicted by $\phi_{\mathrm{bo}}$. In figure $11, \phi_{\mathrm{bo}}$ has been plotted against $W_{\mathrm{p}}$ for various doping densities of the p-region till the effective barrier height $\phi_{\mathrm{bn}}$ reaches its maximum, equal to energy gap $E_{\mathrm{g}}$ (Ag-p-nSi systems). It has been found that $\phi_{\mathrm{bo}}$ has not the same value as the barrier height of the corresponding Schottky device before the inclusion of the inversion layer in it. It may change even by $0.08 \mathrm{eV}$ (from $0.67 \mathrm{eV}$ to $0.75 \mathrm{eV}$ ) towards the high doping densities of the inversion layer. Therefore, the earlier calculations $[14,13]$ based upon the assumption of equality of $\phi_{\mathrm{bog}}$, after fabricating the metal-p-nSi device, to the barrier height of the corresponding Schottky diode without inversion layer, are not so accurate. However, for low dopings
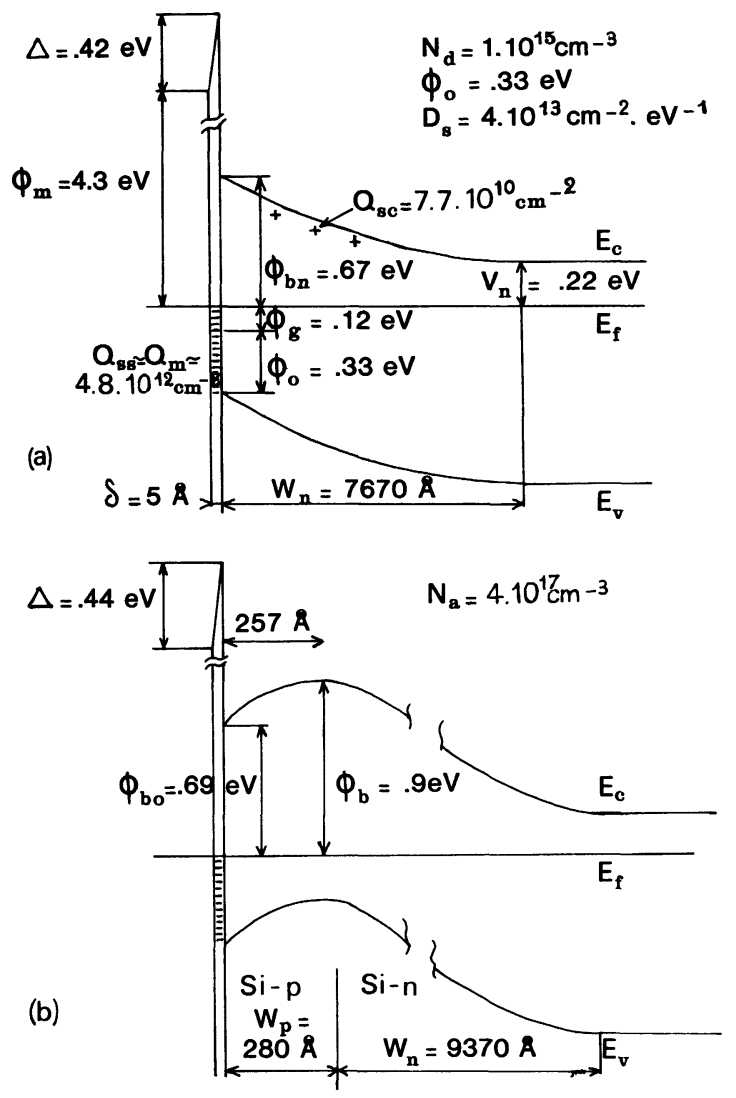

Fig. 14. - Calculated energy band diagram of (a) a simple Ag-nSi Schottky diode; (b) a particular Ag-p-nSi structure.

of the inversion layer this assumption may be tenable (see Fig. 11b).

To test the validity of the above model, it has been applied to Al-p-nSi devices prepared by Card [5] by thermal annealing of simple Al-nSi tunnel structures. In this process an aluminium doped p-region is created between aluminium and $\mathrm{n}-\mathrm{Si}$ by the diffusion of silicon into aluminium and subsequent cooling. $\mathrm{He}$ used different temperatures of annealing and measured the resultant barrier heights by $I-V$ and $C-V$ methods. His results are shown in figure 15 along with the theoretical values calculated according to the present model. The values of the thickness $W_{\mathrm{p}}$ of recrystallised p-region and the corresponding average doping densities $N_{\mathrm{a}}$ have been estimated by the data available in literature $[6,5]$ based upon the assumption of uniformity of p-layer. It is found that there is a good agreement between the present calculated values of barrier heights and those deduced from the experiments of Card [5]. It should be noted here that the height EA (Fig. 7) of the maximum potential point above the Fermi level has been taken to be the barrier height measured by $I-V$ characteristics and the intercept $\mathrm{DB}$ at the potential axis by the extended parabola OAB has been taken to be the barrier height measured by $C-V$ experiments.

As a second example of the validity of the model, it has been applied to the recent experiments of Li et al. [7] 


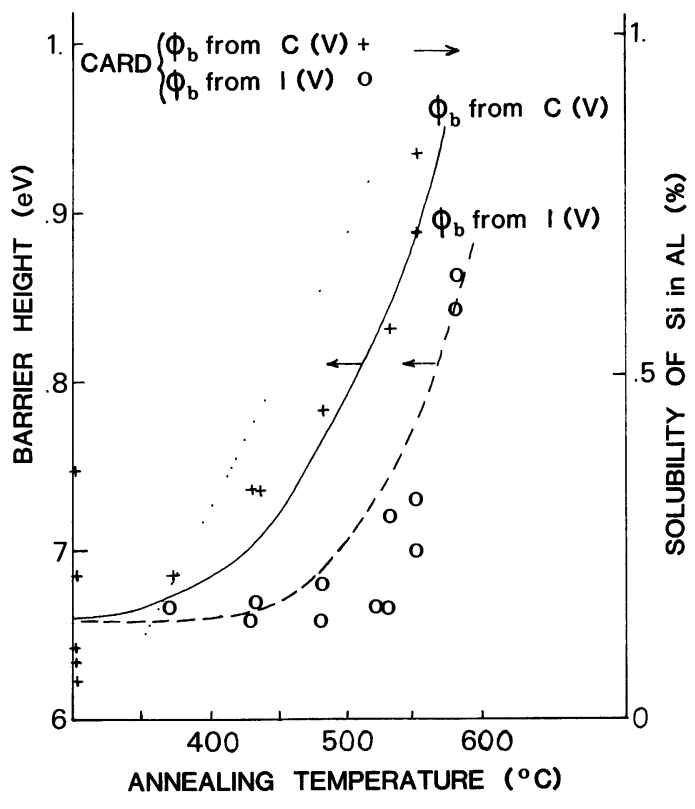

Fig. 15. - Comparison of the barrier height values deduced from Card's experiments [5] and the present work.

on Ti-n-pSi devices fabricated by low energy ionimplantation of phosphorous onto p-Si substrates of (100) orientation. In their experiments, they found that for $N_{\mathrm{d}}$ between $10^{16}$ and $3.1 \times 10^{17} \mathrm{~cm}^{-3}$ and n-layer thickness varying upto $780 \AA$, Ti-pSi device showed barrier heights ranging from $0.6 \mathrm{eV}$ to $0.96 \mathrm{eV}$. Our calculation for the experiments of $\mathrm{Li}$ et al. have been presented in figure 16 together with their experimental results, showing a good fit. The density of surface states at the interface in these experiments has been estimated to suit the barrier height of Ti-pSi
Schottky diode seen without growing the inversion layer which, of course, is subject to the crystal orientation [15].

The small discrepancies between the results based upon the present theory and the experiments may be attributed to the various assumptions made in its formulation. For instance the assumption of abrupt p-n or n-p junctions is not valid in such devices. In annealing experiments the non-uniformity of the thickness of the recrystallized region makes it further untenable. In ion implantation experiments too, the nonuniformity effect in the doping profile is very large.

The inequality :

$$
N_{\mathrm{a}} W_{\mathrm{p}}>N_{\mathrm{d}} W_{\mathrm{n}}
$$

is the condition of existence of a maximum potential point $x_{\mathrm{m}}$ lying in the interval $W_{\mathrm{n}}<x_{\mathrm{m}}<W_{\mathrm{n}}+W_{\mathrm{p}}$ (as shown by the expression (14)). If $N_{\mathrm{a}} W_{\mathrm{p}}=N_{\mathrm{a}} W_{\mathrm{n}}$, $x_{\mathrm{m}}=W_{\mathrm{p}}+W_{\mathrm{n}}$, which implies that the maximum potential is just on the interface at the end of the pregion. If $N_{\mathrm{a}} W_{\mathrm{p}}<N_{\mathrm{d}} W_{\mathrm{n}}$, then $x_{\mathrm{m}}>W_{\mathrm{p}}+W_{\mathrm{n}}$, there is no potential maximum in the p-region, and no increase of the barrier height. However the shape of the band is no more parabolic, which can introduce an error in the barrier height determination from $C(V)$ measurement, where band parabolicity is usually assumed.

From the above discussion it can be concluded that the value of space charge in the inverted region should be above a minimum threshold to keep the peak in the p-region.

Assumption (iv) of section 4, about the independence of surface properties of the semiconductor before and after creating the inversion region to fabricate metal/ p-n or metal/n-p Schottky diodes is an arbitrary one.

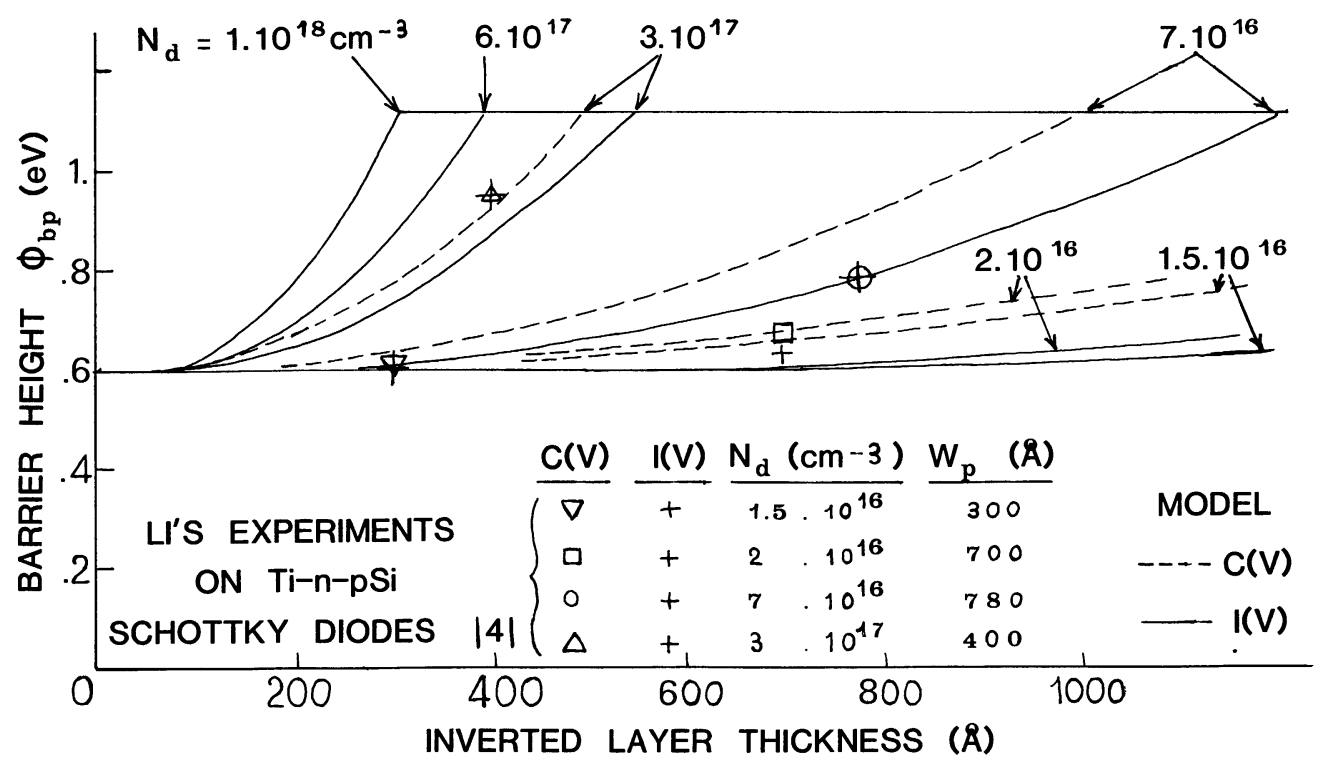

Fig. 16. - Variation of effective barrier height of Ti-n-pSi systems with the thickness of n-region for its various doping densities (Li's experiment [7]). 
However this behaviour is expected from the fact that surface states are essentially a property of the semiconductor/metal couple.

Calculations based upon the present model show that the barrier height of Schottky devices having inversion layers can attain values as high as the energy gap $E_{\mathrm{g}}$ of the semiconductor (Figs. 10,11, 12). However, it should be noted that towards the high values of built-in potential, the assumption of complete ionization of the inversion region (assumption (v) in section 4) may not hold true. The net charge density in this region may not be attributed only to its complete ionization. There will be significant contribution of the positive charge due to holes (in the case of metal-pnSi device) accumulated in the potential well of this region. Therefore, towards the very high values of barrier height, i.e. for the high values of thickness $W_{\mathrm{p}}$ of p-region and its concentration $N_{\mathrm{a}}$, the model may not hold true.

The introduction of the inversion region at the interface has an important effect on the currentvoltage characteristics of the resultant device. The space charge created by the surface layer is opposite to that due to the depleted bulk semiconductor. Consequently, the field at the interface also becomes opposite in sign. If the inversion region thickness is large, the ideality of the device may be damaged. To avoid it, care should be taken to fabricate high barrier height devices by creating shallow surface layers with high doping densities. At the same time, the maximum potential point should be as close to inter- face as possible, to avoid increase in the ideality factor. The electrical characteristics of such devices will be discussed with more detail in a future paper.

\section{Conclusions}

Whether or not Schottky barriers with inversion layers can be suitable alternatives to conventional p-n junctions, for applications such as solar cells, will depend on the electrical and photoelectric properties of these devices, which remain to be evaluated. Presently we can conclude that this modified form of Schottky barrier allows to make the barrier height flexible and able to reach a value of the order of the energy gap of the semiconductor, a behaviour unlike that of $p-n$ junctions. We have also suggested that the stability of such devices is better than that of simple metal-semiconductor systems. Our model has been found to be compatible with the existing experimental data, which are scarce; new experiments should be performed, in particular with implanted inversion layers.

\section{Acknowledgments.}

The author is very much indebted to $M$. Rodot for stimulating discussions on the problems raised by this paper. A useful talk with C. Barret and A. Vapaille is gratefully acknowledged. French « Ministère des Relations Extérieures " and UNESCO are thanked for having made this stay in a CNRS laboratory possible.

\section{References}

[1] Brillson, L. J., J. Phys. Chem. Solids 44 (1983) 703.

[2] Cowley, A. M., Sze, S. M., J. Appl. Phys. 36 (1965) 3213.

[3] BardeEn, J., Phys. Rev. 71 (1947) 717.

[4] Barret, C., Vapaille, A., J. Appl. Phys. 50 (1979) 4217.

[5] CARD, H. C., Inst. Physics Conf. Ser. 22 (1974) 129.

[6] CARD, H. C., IEEE Electron Device Letters, EDL 2 (1981) 149

[7] LI, S. S., KIM, J. S., WANG, K. L., IEEE Trans. Electron. Dev. ED 27 (1980) 1310.

[8] Aina, O., Pande, K. P., J. Appl. Phys. 56 (1984) 1717.
[9] SzE, S. M., Physics of semiconductor devices (Wiley) 1979.

[10] Milnes, A. G., Semiconductor devices and integrated electronics (VNB Press, New York) 1980, p. 100.

[11] El Sayed, M., Pananakakis, G., Kamarinos, G., Solid State Electron. (to be published).

[12] Duong, A. K., Nassilian, A. G., J. Appl. Phys. 57 (1985) 1256.

[13] VAN DER ZIEL, A., Solid State Electron. 20 (1977) 269.

[14] Shannon, J. M., Solid State Electron. 19 (1976) 537.

[15] Gray, P. V., Brown, D. M., Appl. Phys. Lett. 8 (1966) 31. 